\title{
Optimal Fuzzy Controller Mapped from LQR under Power and Torque Constraints
}

\author{
Ping-Ho Chen ${ }^{1}$ and Kuang-Yow Lian ${ }^{2}$ \\ ${ }^{1}$ Department of EE, Tungnan University, Taipei, Taiwan \\ phcamail.tnu.edu.tw \\ ${ }^{2}$ Department of EE, National Taipei University of Technology, Taipei, Taiwan \\ kylian@ntut.edu.tw
}

\begin{abstract}
Dealing with a LQR controller surface subject to power and torque constraints, is an issue of nonlinear problem that is difficult to implement. This paper employs a fuzzy controller surface to replace the LQR surface subject to power and torque constraints by using class stacking, least square and Sugenotype fuzzy inference mode. Through this type of transformation, called "Optimal fuzzy controller mapped from LQR", control of the system remains optimal.
\end{abstract}

Keywords: LQR, FLC, Fuzzy, Sugeno, Optimal, Class stacking.

\section{Linear Quadratic Regulator (LQR)}

The development of optimal control for a MIMO system [1][2] is mature nowadays. Approach of linear quadratic regulator (LQR) offers Kalman gain for state feedback to regulate a MIMO system in state-space form. But subject to power and torque limitation of motor drive, implementation of system becomes difficult using LQR approach. This section introduces some basis for LQR approach for further improvement.

\subsection{LQR Approach}

For a MIMO system in state-space form

$$
\dot{X}=A X+B u
$$

where $\boldsymbol{X} \in R^{n}$ is the state vector. The control $\boldsymbol{u} \in R^{w}$ includes state-feedback torque in motor application, command for desired state output and disturbance that the system might encounter. Control $\boldsymbol{u}$ is thus expressed by

$$
\boldsymbol{u}=-\boldsymbol{K} \boldsymbol{X}+\boldsymbol{u}_{\text {com }}+\boldsymbol{u}_{\text {dist }}
$$

where $\boldsymbol{K}$ : Kalman gain. $\boldsymbol{u}_{\text {dist }}$ : disturbance torque (colored noise). $\boldsymbol{u}_{\text {com }}$ : command for desired state output. Eq. (2) is a general form of input in LQG (Linear Quadratic Gaussian) problems. When $\boldsymbol{u}_{\text {dist }}=\boldsymbol{o}$ and $\boldsymbol{u}_{\text {com }} \neq \boldsymbol{o}$, the system becomes a tracking problem expressed by 


$$
\dot{\boldsymbol{X}}=(\boldsymbol{A}-\boldsymbol{B} \boldsymbol{K}) \boldsymbol{X}+\boldsymbol{B} \boldsymbol{u}_{\text {com }}
$$

At steady state it satisfies

$$
\boldsymbol{O}=(\boldsymbol{A}-\boldsymbol{B} \boldsymbol{K}) \boldsymbol{X}+\boldsymbol{B} \boldsymbol{u}_{\text {com }}
$$

In case $\boldsymbol{u}_{\text {com }}=\boldsymbol{O}$ in Eq. (4), the system becomes a regulator problem further called"linear quadratic regulator (LQR)", if we select a gain $\boldsymbol{K}$, called Kalman gain such that $\boldsymbol{u}=-\boldsymbol{K} \boldsymbol{X}$ to minimize the cost $\boldsymbol{J}$ in quadratic form as

$$
\begin{gathered}
J=\frac{1}{2} \boldsymbol{X}^{\mathrm{T}}(T) \boldsymbol{S}(T) \boldsymbol{X}+\int_{0}^{T}\left[\frac{1}{2}\left(\boldsymbol{X}^{\mathrm{T}} \boldsymbol{Q} \boldsymbol{X}+\boldsymbol{u}^{\mathrm{T}} \boldsymbol{R} \boldsymbol{u}\right)\right. \\
\left.+\lambda^{\mathrm{T}}(\boldsymbol{A} \boldsymbol{X}+\boldsymbol{B} \boldsymbol{u}-\dot{\boldsymbol{X}})\right] d t
\end{gathered}
$$

where $Q$ : dynamic state weighting, $R$ : control weighting $S$ : terminal state weighting and $\lambda$ : Lagrange multiplier. After several algebra operations to minimize the cost, we have the following Hamiltonian matrix presenting as the transition matrix in Eq. (6):

$$
\begin{gathered}
{\left[\begin{array}{c}
\dot{\boldsymbol{X}} \\
\dot{\lambda}
\end{array}\right]=\left[\begin{array}{cc}
\boldsymbol{A} & -\boldsymbol{B R}^{-1} \boldsymbol{B}^{\mathrm{T}} \\
-\mathrm{Q} & -\boldsymbol{A}^{\mathrm{T}}
\end{array}\right]\left[\begin{array}{l}
\boldsymbol{X} \\
\lambda
\end{array}\right]} \\
\lambda(t)=\boldsymbol{S}(t) \boldsymbol{X}(t) \\
\lambda(T)=\boldsymbol{S}(T) \boldsymbol{X}(T) \\
\boldsymbol{U}(t)=-\boldsymbol{R}^{-1} \boldsymbol{B}^{\mathrm{T}} \lambda(t)=-\boldsymbol{R}^{-1} \boldsymbol{B}^{\mathrm{T}} \boldsymbol{S}(t) \boldsymbol{X}(t)=-\boldsymbol{K}(t) \boldsymbol{X}(t)
\end{gathered}
$$

The steady gain is

$$
\boldsymbol{K}=\lim _{t \rightarrow \infty} \boldsymbol{K}(t)=\boldsymbol{R}^{-1} \boldsymbol{B}^{\mathrm{T}} \lim _{t \rightarrow \infty} \boldsymbol{S}(t)=\boldsymbol{R}^{-1} \boldsymbol{B} \boldsymbol{\psi}_{21}\left(\boldsymbol{\psi}_{11}\right)^{-1}
$$

where $\boldsymbol{\psi}_{21}$ and $\boldsymbol{\psi}_{11}$ are the first-column block matrix of $\boldsymbol{\Psi}$ which is the modal form of the Hamiltonian matrix in Eq. (6).

\subsection{Constraints of LQR Control}

While implementing the optimal control $\boldsymbol{u}$ aforementioned, we always meet difficulty when subject to constraints of torque limit $\boldsymbol{u}_{l}$ and power limit $p_{l}$. The torque constraint, caused by stall current in motor application, is expressed by

$$
\boldsymbol{u} \leq \boldsymbol{u}_{l}, \boldsymbol{u}_{l}=k_{i} \boldsymbol{i}_{s}
$$

where $k_{i}$ : current constant of motor. $\boldsymbol{i}_{s}$ : stall current of motor. As usual the control $\boldsymbol{u}$ is associated with states of position and rate. Mechanical power $p_{m}$, constrained by maximum power $p_{l}$ of motor, is represented by 


$$
p_{m}=\boldsymbol{u}^{\mathrm{T}} \dot{\boldsymbol{\theta}} \leq p_{l}
$$

where $\dot{\boldsymbol{\theta}}$ is rate-related state vector.

\section{Class Stacking}

From Eq. (2), the LQR control is $\boldsymbol{u}=-\boldsymbol{K} \boldsymbol{X}=\left[u_{1} u_{2} \cdots u_{w}\right]^{t}$ where $\boldsymbol{K}=\left[k_{j i}\right] \in R^{w \times n}$ and $u_{j}=-\sum_{i=1}^{n} k_{j i} x_{i}$ for $j=1, \cdots, w$. Since each component $u_{j}$ of $\boldsymbol{u}$ will be solved one by one by the same approach of class stacking, $u_{j}$ will be replaced by $u=\sum_{i=1}^{n} k_{i} x_{i}$ for illustration without loss of generality. Obviously for $j^{\text {th }}$ component $u_{j}$ of $\boldsymbol{u}, k_{i}=-k_{j i}$. The form of $u=\sum_{i=1}^{n} k_{i} x_{i}$ is a hyper plane of $n$-D. All the states $x_{i}$ for $i=1, \cdots, n$ are sorted out into two classes. One class, having $m$ states, is position-related and piled up by

$$
v_{1}=\sum_{i=1}^{m} k_{i} x_{i}
$$

and the other class, having $n-m$ states, is rate-related and piled up by

$$
v_{2}=\sum_{i=m+1}^{n} k_{i} x_{i}
$$

Then

$$
u=v_{1}+v_{2}
$$

The torque constraint is

$$
v_{1}+v_{2}=u \leq u_{l}
$$

The power constraint $u v_{2} \leq p_{l}$ from Eq. (12) can be rewritten by

$$
\left(v_{1}+v_{2}\right) v_{2} \leq p_{l}
$$

Eq. (13) $\sim(15)$ thus reduce the system from $n$-D into 3 -D with variables $v_{1}$ and $v_{2}$. Eq. (16) indicates a plane $v_{1}+v_{2}=u$ with known LQR control $u$ but upper bounded by $u_{l}$. Eq. (17) indicates a nonlinear constraint. The process, called "class stacking", thus make it feasible to plot a hyper plane of $n$-D in terms of 3-D. An example using computer-aided plot is given as follows. A LQR control $u$, with number of total states $n=5$ and number of position-related states $m=3$, is expressed by 


$$
u=\sum_{i=1}^{5} k_{i} x_{i}=v_{1}+v_{2}, v_{1}=\sum_{i=1}^{3} k_{i} x_{i}, v_{2}=\sum_{i=4}^{5} k_{i} x_{i}
$$

where $\left|x_{i}\right| \leq 1$ (normalized) and $k_{i}=i$ for $i=1, \cdots, 5$. Obviously maximum of $v_{1}$ is 6 and maximum of $v_{2}$ is 9 , subject to constraints: $v_{1}+v_{2} \leq 12$ (Upper bound) and $\left(v_{1}+v_{2}\right) v_{2} \leq 75$ (Inequality constraint).

By using computer-aided plot [3], Fig. 1(a)-(d) shows a sequence of transformations from LQR surface to cases of constraints are obtained. Fig. 1(a) shows the interception of a LQR surface, a torque-constrained surface and a hyperboloid due to power constraint. Fig. 1(b) shows LQR surface bounded by all constraints.
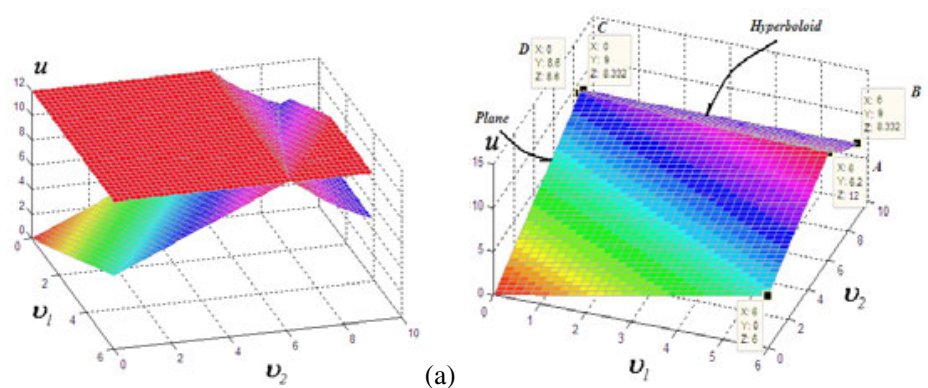

(b)

Fig. 1. Computer-aided plot (a) Interception of LQR plane, torque constraint plane and power constraint hyperboloid (b) LQR surface bounded by all constraints

\section{Fuzzy Logic Controller (FLC)}

Although the approach of class stacking reduces the hyper plane from the LQR controller of $n$-D into a bounded and constrained plane in $3-\mathrm{D}$, the $3-\mathrm{D}$ controller surface shown in Fig. 1.(b) is still nonlinear. Therefore we may employ a fuzzy-logiccontroller (FLC) to implement this 3-D controller surface shown in Fig. 1.(b).

\subsection{Fuzzy Inference System (FIS)}

A fuzzy logic controller (FLC) [4][5][6] is configured by processing a sequence of fuzzification, inference and defuzzification as follows. The fuzzy inference system (FIS) to be selected is Sugeno model [7] given in Fig. 2 with $v_{1}$ and $v_{2}$ as input fuzzy variables and the control $u$ as known output variable which is bounded, constrained and expressed by plane $s 1: u=v_{1}+v_{2}$, plane $s 2: u=12$ (Upper bound) and hyperboloid: $u v_{2} \leq 75$ (Inequality constraint).

\subsection{Fuzzification}

Input fuzzy variables are $v_{1}$ and $v_{2} . v_{1}$ has five triangle membership functions, equally partitioned and spaced as shown in Fig. 2. Membership functions are defined 
by linguistic terms, i.e. S, SM, M, MB and B. Output variable is the control $u$ that has five membership functions, i.e. $\mathrm{S}+\mathrm{SM}+\mathrm{M}+\mathrm{MB}+$ and $\mathrm{B}$ as shown in Fig. 2.
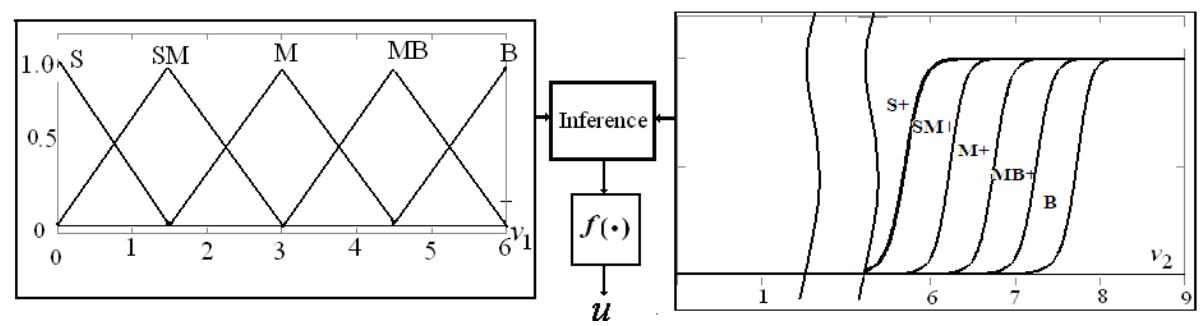

Fig. 2. Fuzzy inference system (FIS) using Sugeno model

\subsection{Inference}

Output variable is the control $u$ that is composed of plane $s 1$ and a hyperboloid. The plane $s 1$ is $u=v_{1}+v_{2}$. The hyperboloid $A B C D$ in Fig. 1(b) is approximately replaced by another plane in Fig. 3.(a), named $s 2$ and obtained by least square with

$$
\begin{gathered}
s 2: u=\Lambda\left[\begin{array}{lll}
v_{2} & v_{1} & 1
\end{array}\right]^{\mathrm{T}} \\
\Lambda=\left[\begin{array}{lll}
-0.8025 & 0.2566 & 14.7253
\end{array}\right]
\end{gathered}
$$

$\boldsymbol{\Lambda}$ is obtained by

$$
\boldsymbol{\Lambda}^{\mathrm{T}}=\left(\boldsymbol{Y}^{\mathrm{T}} \boldsymbol{Y}\right)^{-1}\left(\boldsymbol{Y}^{\mathrm{T}} \boldsymbol{Z}\right)
$$

with

$$
\boldsymbol{Y}=\left[\begin{array}{lll}
v_{2 A} & v_{l_{A}} & 1 \\
v_{2 B} & v_{l_{B}} & 1 \\
v_{2 C} & v_{I_{C}} & 1 \\
v_{2 D} & v_{I_{D}} & 1
\end{array}\right], \boldsymbol{Z}=\left[\begin{array}{l}
u_{A} \\
u_{B} \\
u_{C} \\
u_{D}
\end{array}\right]
$$

The selected $\boldsymbol{\Lambda}$ is able to minimize error $\|\boldsymbol{Z}-\boldsymbol{Y} \boldsymbol{\Lambda}\|$.

After finishing the definition of linear planes, we are able to develop the inference that includes ten rules. The former half governs the surface $s 2$ and the latter half governs the surface $s 1$. Rules are written as follows.

1. If ( $v_{1}$ is $\left.\mathrm{S}\right)$ and $\left(v_{2}\right.$ is $\left.\mathrm{B}\right)$ then $(u$ is $s) ; 2$. If $\left(v_{1}\right.$ is $\left.\mathrm{SM}\right)$ and $\left(v_{2}\right.$ is $\left.\mathrm{MB}+\right)$ then $(u$ is $s 2)$

3. If ( $v_{1}$ is $\left.\mathrm{M}\right)$ and $\left(v_{2}\right.$ is $\left.\mathrm{M}+\right)$ then ( $u$ is $\left.s\right)$; 4 . If $\left(v_{1}\right.$ is $\left.\mathrm{MB}\right)$ and $\left(v_{2}\right.$ is $\left.\mathrm{SM}+\right)$ then $(u$ is $s 2)$

5. If ( $v_{1}$ is $\left.\mathrm{B}\right)$ and $\left(v_{2}\right.$ is $\left.\mathrm{S}+\right)$ then $(u$ is $s 2) ; 6$. If $\left(v_{1}\right.$ is $\left.\mathrm{S}\right)$ and ( $v_{2}$ is not $\left.\mathrm{B}\right)$ then $(u$ is $s 1)$

7. If ( $v_{1}$ is $\left.\mathrm{SM}\right)$ and ( $v_{2}$ is not $\mathrm{MB}+$ ) then ( $u$ is $\left.s 1\right)$; 8 . If ( $v_{1}$ is $\left.\mathrm{M}\right)$ and ( $v_{2}$ is not $\mathrm{M}+$ ) then ( $u$ is $s 1)$; 9. If $\left(v_{1}\right.$ is $\left.\mathrm{MB}\right)$ and $\left(v_{2}\right.$ is not $\left.\mathrm{SM}+\right)$ then $(u$ is $s 1) ; 10$. If $\left(v_{1}\right.$ is $\left.\mathrm{B}\right)$ and $\left(v_{2}\right.$ is not $\mathrm{S}+)$ then $(u$ is $s 1)$. 


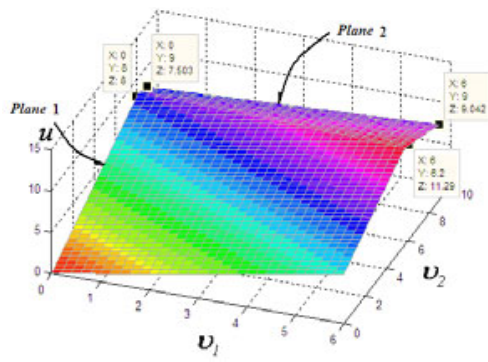

(a)

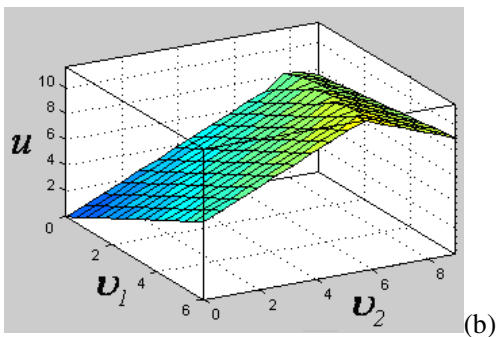

Fig. 3. (a) Hyperboloid is approximately replaced by plane $s 2$ (b) Rule surface after fuzzy defuzzification

\subsection{Defuzzification}

A rule surface after defuzzification is generated as shown in Fig. 3(b) which is observed to be an approximation of Fig. 3.(a).

\section{Conclusion}

LQR control surface in hyperspace, subject to power and torque constraints, is difficult to implement. This paper employs class stacking to transform LQR surface in hyper space into a surface in 3-D and further applies a Sugeno-type fuzy logic controller to plot the 3-D nonlinear surface. This approach makes LQR feasible and visible.

\section{References}

1. Lewis, F.L.: Applied Optimal Control \& Estimation. Prentice Hall, Englewood Cliffs (1992)

2. Burl, J.B.: Linear Optimal Control. Addison-Wesley, Reading (1999)

3. Matlab: toolbox: Fuzzy (2010)

4. Zadeh, L.A.: The concept of a linguistic variable and its application to approximate reasoning. Parts 1, 2, and 3. Information Sciences 8, 199-249, 8, 301-357, 9, 43-80 (1975)

5. Mamdani, E.H., Assilian, S.: An Experiment in Linguistic Synthesis with Fuzzy Logic Controller. Int. J. Man Mach. Studies 7(1), 1-13 (1975)

6. Mamdani, E.H.: Advances in the linguistic synthesis of fuzzy controllers. International Journal of Man-Machine Studies 8, 669-678 (1976)

7. Sugeno, M.: Fuzzy measures and fuzzy integrals: a survey. In: Gupta, M.M., Saridis, G.N., Gaines, B.R. (eds.) Fuzzy Automata and Decision Processes, pp. 89-102. North-Holland, NY (1977) 